\title{
Tool-use learning by common marmosets (Callithrix jacchus)
}

\author{
Yumiko Yamazaki · Chieko Echigo • Masakado Saiki · \\ Masayuki Inada $\cdot$ Shigeru Watanabe $\cdot$ Atsushi Iriki
}

Received: 11 March 2011 / Accepted: 15 June 2011 / Published online: 29 June 2011

(C) The Author(s) 2011. This article is published with open access at Springerlink.com

\begin{abstract}
One of the most critical and common features of tool use is that the tool essentially functions as a part of the body. This feature is likely rooted in biological features that are shared by tool users. To establish an ideal primate model to explore the neurobiological mechanisms supporting tool-use behaviours, we trained common marmosets, a small New World monkey species that is not usually associated with tool use, to use a rake-shaped tool to retrieve food. Five naive common marmosets were systematically trained to manipulate the tool using a 4-stage, step-by-step protocol. The relative positions of the tool and the food were manipulated, so that the marmosets were required to (1) pull the tool vertically, (2) move the tool horizontally, (3) make an arc to retrieve a food item located behind the tool and (4) retrieve the food item. We found considerable individual differences in tool-use technique; for example, one animal consistently used a unilateral hand movement
\end{abstract}

Electronic supplementary material The online version of this article (doi:10.1007/s00221-011-2778-9) contains supplementary material, which is available to authorized users.

Y. Yamazaki $\cdot$ S. Watanabe

The Graduate School of Sociology, Keio University,

201 Mita Toho Building, 3-1-7 Mita, Minato-ku,

Tokyo 108-0073, Japan

Y. Yamazaki $(\bowtie) \cdot$ M. Saiki $\cdot$ M. Inada $\cdot$ A. Iriki

Laboratory for Symbolic Cognitive Development,

RIKEN Brain Science Institute, 2-1 Hirosawa,

Wako-shi, Saitama 351-0198, Japan

e-mail: yumyam@brain.riken.jp

C. Echigo $\cdot$ S. Watanabe

Department of Psychology, Faculty of Letters, Keio University,

2-15-45 Mita, Minato-ku, Tokyo 108-8345, Japan for all of the steps, whereas the others $(n=4)$ used both hands to move the tool depending on the location of the food item. After extensive training, all of the marmosets could manipulate the rake-shaped tool, which is reported in this species for the first time. The common marmoset is thus a model primate for such studies. This study sets the stage for future research to examine the biological mechanisms underlying the cognitive ability of tool use at the molecular and genetic levels.

Keywords Common marmoset $\cdot$ Hand preference .

Tool use

\section{Introduction}

Tool use is widespread among primates and is seen in both Old and New World monkeys. Chimpanzees (Pan troglodytes) in Africa use a different combination of stones as anvils and hammers to crack nuts (Matsuzawa 1994). Stone tool use has also been observed in wild cebus monkeys (Cebus libidinosus) in South America (Fragaszy et al. 2004; Moura and Lee 2004) and long-tailed macaques (Macaca fascicularis) in Asia (Malaivijitnond et al. 2007). The type of tool used by these primates is called a "motor tool" (Asano 1994; Yamazaki et al. 2009) and can enhance the animals' motor abilities. Considering that Old and New World monkeys evolutionarily divided more than 33 million years ago (Glazko and Nei 2003), it is possible that they developed different tool-use behaviours due to ethological factors in their habitats. In fact, the motor tool is well developed among animals, such as crows (e.g. Hunt 1996).

However, evidence of spontaneous tool use is very limited in both Japanese macaques (Macaca fuscata) (but see 
Leca et al. 2010) and common marmosets (C. jacchus). However, research has shown that Japanese macaques can be trained to use rake-shaped tools (e.g. Iriki et al. 1996) and to develop complex tool-use behaviours (Hihara et al. 2003; Yamazaki et al. 2009). In the training procedures applied in our studies, the spatial relationship between tools and food items was carefully arranged to allow the animals to move the tool appropriately to get the food items. Using these procedures, naive Japanese macaques quickly learned to manipulate the rake-like tools within 2 weeks (Quallo et al. 2009; Yamazaki et al. 2009). This suggests that primates that do not use tools in the wild may be equipped with brain mechanisms that support the learning of novel tool-use behaviours, if given the appropriate training.

In the present study, we sought to broaden our understanding of how tool-use behaviours can be manipulated in the common marmoset, a New World monkey. There has been no report of tool usage in common marmosets in the wild. If common marmosets can successfully learn to use tools, as macaques can, then the common marmoset could be used to further investigate the molecular basis for the acquisition of novel behaviours involving changes in neural circuitries and morphologies (e.g. Iriki et al. 1996; Ishibashi et al. 2002; Hihara et al. 2006; Quallo et al. 2009) in transgenic marmosets (Sasaki et al. 2009).

Previous reports have suggested that marmosets can discriminate between objects based on the presence or absence of a physical association and can learn relevant aspects of the objects' spatial relationships (Yamazaki et al. 2011). In a task used to examine the understanding of physical associations, an object is placed out of a subject's reach and rests on a cloth that is within the subject's reach. The test entails observing whether the subject pulls the cloth supporting the object to obtain the object, thereby demonstrating an understanding that the object on the cloth will move closer when the supporting cloth is pulled. The subject is required to understand that the object is resting on the supporting cloth and would not be pulled closer if it was only in contact with the cloth or covered by the cloth. In the case of the rake, which was used as a tool in the present study, a similar understanding of the physical relation between objects is required; in other words, the attractive object (e.g. the food item) is contacted and supported by the rake tip on the table. The objective of the support problem is to assess the subjects' ability to discriminate between alternatives depending on the support. Similarly, the rake problem requires the subjects to understand the relation of the support between two objects and to modify this relation by voluntarily manipulating the tool. Thus, the successful training of tool use would support the notion that these animals can comprehend support from both the production (tool use) and recognition (discrimination of support) perspectives.
Table 1 Age of the subjects at the beginning of the experiment

\begin{tabular}{llllll}
\hline Subject & 1 & 2 & 3 & $\mathrm{k} 1$ & $\mathrm{k} 3$ \\
\hline Age & 2 y $4 \mathrm{~m}$ & 1 y $3 \mathrm{~m}$ & 1 y $3 \mathrm{~m}$ & 2 y $2 \mathrm{~m}$ & 1 y $9 \mathrm{~m}$ \\
\hline
\end{tabular}

$y$ Year, $m$ month

\section{Materials and methods}

\section{Subjects}

Five adult female common marmosets (subject 1, 2, 3, k1 and $\mathrm{k} 3$ ), weighing approximately $310-460 \mathrm{~g}$, were used in the experiments. The animals were laboratory-born, and their ages ranged from 1 y $3 \mathrm{~m}$ to 2 y $4 \mathrm{~m}$ at the beginning of the experiment (see Table 1). Subjects 1 and 3 were raised by human caretakers. The other 3 animals were raised by their parents at the RIKEN Brain Science Institute in Wako (hereafter referred to as RIKEN). All animals were housed individually in a breeding room on a 12-h lightdark cycle. The temperature and humidity in the breeding room were kept, on average, at $27^{\circ} \mathrm{C}$ and $50 \%$, respectively. Testing sessions were always conducted a few hours before daily feeding. The animals were fed regularly, and water was freely available in their cages.

This study complied with the current laws of Japan, including the Act on Welfare and Management of Animals. All experimental procedures and handling methods were performed in accordance with the "Guidelines for Conducting Animal Experiments" of RIKEN. The experiments were approved by the Animal Experiment Committee at RIKEN.

\section{Apparatus}

The experimental chamber was $42(\mathrm{~h}) \times 34(\mathrm{w}) \times 35(\mathrm{~d}) \mathrm{cm}$ and was made of stainless steel. At the bottom of the front panel, there was an aperture $[26(\mathrm{w}) \times 2(\mathrm{~h}) \mathrm{cm}]$ through which the subjects could extend their forearms and hands to move the tool to retrieve food items (described below). The aperture was shaped like a comb with $2 \times 5 \mathrm{~cm}$ teeth. The experimental materials, such as the rake-shaped tool and the food items, were placed on a black acrylic table [9.5 (h) $\times 35(\mathrm{w}) \times 24(\mathrm{~d}) \mathrm{cm}$ ], which was connected to the experimental chamber through the aperture. The height of the table surface was $5.7 \mathrm{~cm}$ above the floor of the experimental chamber, so that the subjects could manipulate the rake in a sitting position.

The tool was shaped like a rake, with a $4.0(\mathrm{w}) \mathrm{cm} \times$ $2.5(\mathrm{~h}) \mathrm{cm}$ white acrylic tip connected to an aluminium rod, $7.0 \mathrm{~cm}$ length in total (see Fig. 1a). The tip was connected to the rod with dental plastic and was painted light green, so 


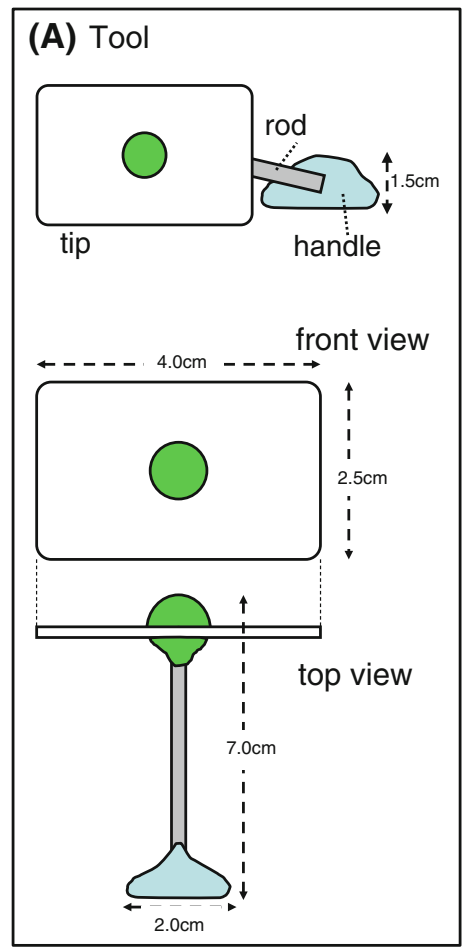

Fig. 1 a Rake-shaped tool used by common marmosets in the training sessions. The tool has a $4 \times 2.5 \mathrm{~cm}$ rectangle tip connected to the rod with dental plastic, which was painted light green, so that it would be easily detected by tracking software. The handle was attached to the end of the shaft, so that it could be easily grasped by the subjects. b Top view of the tool and the food item in different positions for the 4 training stages. The training protocol consisted of 4 stages. Each stage consisted of several steps, as indicated by the Arabic number next to the food item (in orange). In Stage 1, the food item was placed inside the tool tip. It was placed $1 \mathrm{~cm}$ from the edge of the tool tip, with 0,1 and $2 \mathrm{~cm}$ vertical distance for steps 1,2 and 3, respectively. The food was placed on the edge of the tip in step 4. In Stage 2, the food items were placed $0,0.5,1$ and $2 \mathrm{~cm}$ horizontally apart from the edge

it would be easily detected by tracking software. As described below, the size of the tool tip was reduced to $4.0 \mathrm{~cm}(\mathrm{w}) \times 1.5 \mathrm{~cm}(\mathrm{~h})$ in Stage 4 of the tool training protocol. There was a handle, made of dental plastic, on the edge of the rod $[2.0 \mathrm{~cm}(\mathrm{w}) \times 1.5 \mathrm{~cm}(\mathrm{~d})]$, so that the marmosets could manipulate it to move the tool to the desired position.

Pieces of fruit-flavoured sweet jelly (coloured red, green and yellow) of roughly $0.5 \mathrm{~cm}$ in length were used as reinforcers for the marmosets. The colour of the food item had no relation to any specific condition; they were randomly presented to the marmosets.

Because the subjects were quite nervous when they were alone in the experimental chamber, we placed another marmoset in a cage $[27.5(\mathrm{~h}) \times 23.0(\mathrm{w}) \times 42.0(\mathrm{~d}) \mathrm{cm}]$ near the experimental chamber. The companion animal could see the experimental marmoset; however, it could not see the details of the experimental conditions because the cage was placed in a lower position than the experimental chamber. of the tool tip in steps 1, 2, 3 and 4, respectively. In this stage, the vertical distance from the tool tip to the food was $1 \mathrm{~cm}$. In Stage 3, the food items were placed on the opposite side of the tool tip. There were four steps in Stage 3, with increasing vertical distances from the edge of the tool $(0,0.5,1$ and $2 \mathrm{~cm}$, respectively). The horizontal distance was set at $1 \mathrm{~cm}$. In Stage 4, the food item was placed behind and above the tool tip. The horizontal distance relative to the tool edge was 0 (at the edge), 1 and $2 \mathrm{~cm}$ in steps 1, 2 and 3, respectively. The vertical distance from the tool tip was set at $1 \mathrm{~cm}$. Note that the conditions where the food items were placed to the right of the tool were trained first for subjects 1, 2 and 3 in Stages 1 and 2. The left and the right food positions were concurrently trained for subjects $\mathrm{k} 1$ and $\mathrm{k} 3$

During the experimental sessions, the subject's behaviour was recorded using video cameras (HDR-HC9; Sony, Tokyo, Japan) that captured side views (from the left) and top views. These videos were integrated into one output through a multiviewer unit (MV-400, FOR-A). The videotapes were used to count the number of successful trials. In addition, specialised software (Ethovision 3.0, Noldus) tracked the tool trajectory in the video recordings.

\section{Procedure}

To habituate the subjects to the experimental situation and set-up, we first transferred them from the breeding room to the experimental chamber using a carrying cage and then fed them jelly for several days. Once they were consistently coming to the experimenter's hand to retrieve the food, the experimenter introduced the rake on the table and put a piece of food on its tip to encourage the animals to pull the rake to get the food item. After the animals repeatedly 
pulled the rake with the food, the first step of the training protocol was initiated (as described below).

\section{Training protocol}

The authors developed a step-by-step training protocol, which was specifically designed for use with the marmosets. The protocol had four main stages; the relative positions of the food item and the tool varied across the stages. Figure $1 \mathrm{~b}$ is a top view of the tool and the food item, showing the difference in the positional relationship between them in these stages. Each stage contained several steps (indicated by the Arabic number near the food items in Fig. 1b), which varied in terms of the distance that the subjects had to move the tool. In Stage 1, the food item was placed at the side of the tool tip closest to the subjects, so that the subjects were rewarded if they pulled the tool straight. In Stage 2, they were required to move the tool to the left or right in addition to pulling the tool forward. In Stages 3 and 4, they had to learn to move the tool forward from the original position to get the food item, which was placed far away from the tip of the tool.

\section{Stage 1}

Figure $1 \mathrm{~b}$ shows a schematic representation of the spatial relation between the tool and the food item in each stage of the training. In Stage 1, the food item was placed inside of the tool tip. If the animal pulled the tool forward, then the food item was likely to be retrieved. The vertical distance between the tool tip and the food item was $0 \mathrm{~cm}$ (i.e. the food item was in contact with the tool tip) in step 1 of Stage 1 (for convenience, step 1 of Stage 1 is described as "Stage 1-1" hereafter), $1 \mathrm{~cm}$ in Stage 1-2 and $2 \mathrm{~cm}$ in Stage 1-3. In these steps, the horizontal position of the food item was $1 \mathrm{~cm}$ from the edge of the tool tip. In Stage 1-4, the vertical position of the food item was $1 \mathrm{~cm}$ from the tool tip, and the horizontal position was $0 \mathrm{~cm}$ (i.e. just beneath the edge of the tip).

\section{Stage 2}

In this stage, the animals were required to pull the tool forward and to the left or right to cover areas where the tool tip was not accessible with a straight pulling movement. There were four steps in Stage 2, with increasing horizontal distances from the edge of the tool to the food item $(0,0.5,1$ and $2 \mathrm{~cm}$ in Stage 2-1, 2-2, 2-3 and 2-4, respectively). The vertical distance from the edge of the tool was set at $1 \mathrm{~cm}$.

\section{Stage 3}

In Stage 3, the subjects were required to push the tool vertically. There were four steps in Stage 3, with increasing vertical distances from the edge of the tool to the food item $(0-2 \mathrm{~cm})$. The horizontal distance was set at $1 \mathrm{~cm}$.

\section{Stage 4}

In the final stage of the training, the subjects were required to retrieve the food item from behind the tool tip. In the first step of the stage, the food item was placed $1 \mathrm{~cm}$ behind and above the tool tip, at the same position as the edge of the tool tip. Then, the horizontal distance relative to the tool edge was increased to 1 and $2 \mathrm{~cm}$ in steps 2 and 3, respectively. The vertical distance from the tool tip was set at $1 \mathrm{~cm}$ in both steps. In steps 2 and 3, the food item was completely hidden by the tool tip at the beginning of the trial; therefore, the subjects were occasionally reluctant to move the tool and retrieve the food item. Thus, during these steps, we reduced the upper area of the tool tip to $4 \mathrm{~cm} \times 1.5 \mathrm{~cm}$ to make it easier for the animals to find the food item behind the tool tip. If they were reluctant to work, we repeatedly showed them the food item and then put the tool in place after we confirmed that the animals were interested in the food item.

We applied two different sequences of training steps during Stages 1 and 2. For subjects 1, 2 and 3, the food items were placed to the right of the tool until Stage 2. In this training sequence, Stages 1-1, 1-3 and 1-4, where the food was to the left of the tool, were omitted. For subject 2, we additionally omitted Stage 1-2 left and 2-1 left. The animals took a relatively long time to master these conditions; therefore, we tried to apply the protocols by which they were trained to retrieve the food item on the left and the right concurrently for subjects $\mathrm{k} 1$ and $\mathrm{k} 3$. In this modified protocol, if the subject met the acquisition criterion when the food item was placed on the left, we did not move on to the next step but waited until they met the criterion on the right in the same training step. After the conditions on both sides were mastered, then the subjects moved on to the next training step. The numbers of trials with food on the left or right of the tool was nearly equal within each session. All of the animals were trained following the same procedure for Stages 3 and 4. Although some conditions were not present in the early protocol (e.g. steps 1, 3 and 4 on the left for Stage 1), the basic spatial plan for the placement of the food items relative to the tool was the same for all animals.

Throughout the experimental sessions, one experimenter in front of the panel presented the food item and the tool on the table in front of the animals. Daily training sessions lasted up to $20 \mathrm{~min}$. The number of trials executed in a daily session varied greatly among subjects and among training days, especially at the beginning of Stage 1. The subjects completed more than 70 trials/day at maximum, but there were some sessions during which they would not 

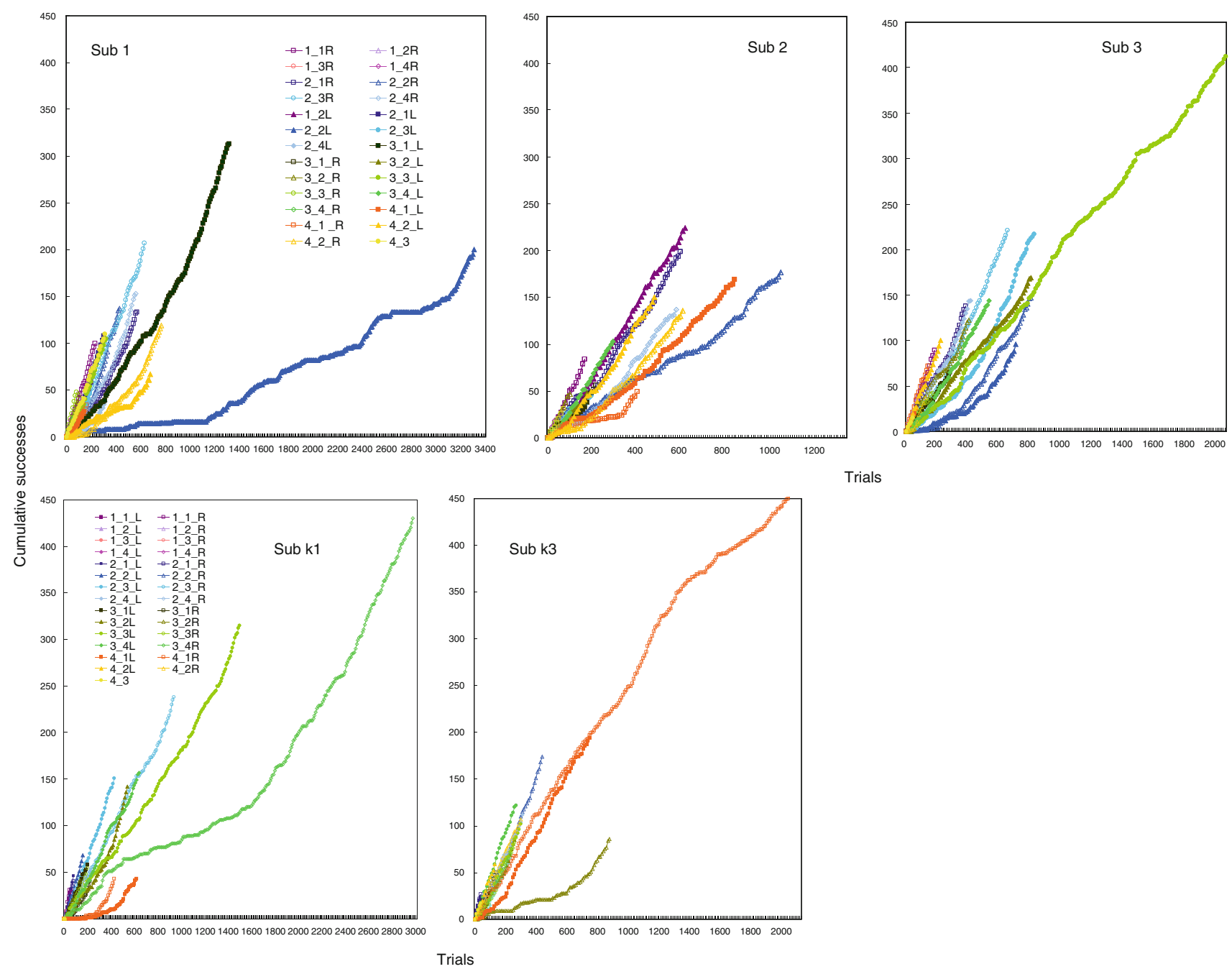

Fig. 2 Cumulative successes in each training step. The steps that required the largest number of trials to achieve mastery varied between subjects

work at all. There was no daily trial assignment that the subjects had to complete; the number of trials they engaged in was completely dependent on their motivation to participate in the experiment. After a subject ate the food item, the next trial started. A successful performance was defined as the retrieval of the food item by the animals without any help from the experimenter. A trial in which the animals repeatedly grasped the tool handle to manipulate the tool was also considered successful. The acquisition criterion to move on to the next training step was defined as 5 consecutive successful trials. During training, the subjects were not forced to use a particular hand/arm for tool use; they freely chose which hand/arm to use. If the subject was reluctant to work, especially in the early phases of a new step, we occasionally reintroduced the preceding conditions to motivate them to participate.

\section{Results}

All subjects were successfully trained to use the tool and finished the final step of the training protocol (Stage 4-3 in Fig. 1). The time course of acquisition in each step varied considerably between subjects and training steps (Fig. 2). The step that required the largest number of trials to achieve mastery also varied by subject: Stage 2-2 on the left for subject 1 (3,290 trials), Stage 2-2 on the right for subject 2 (1,108), Stage 3-3 on the left for subject 3 $(2,048)$, Stage $3-4$ on the right for subject k1 $(2,967)$ and Stage $4-1$ on the left for subject k3 $(2,112)$.

Considerable individual differences were found in the number of trials required to reach the acquisition criterion, as shown in Fig. 3a. The total numbers of trials needed to complete the training were $11,381,8,667,8,980,9,539$ and 


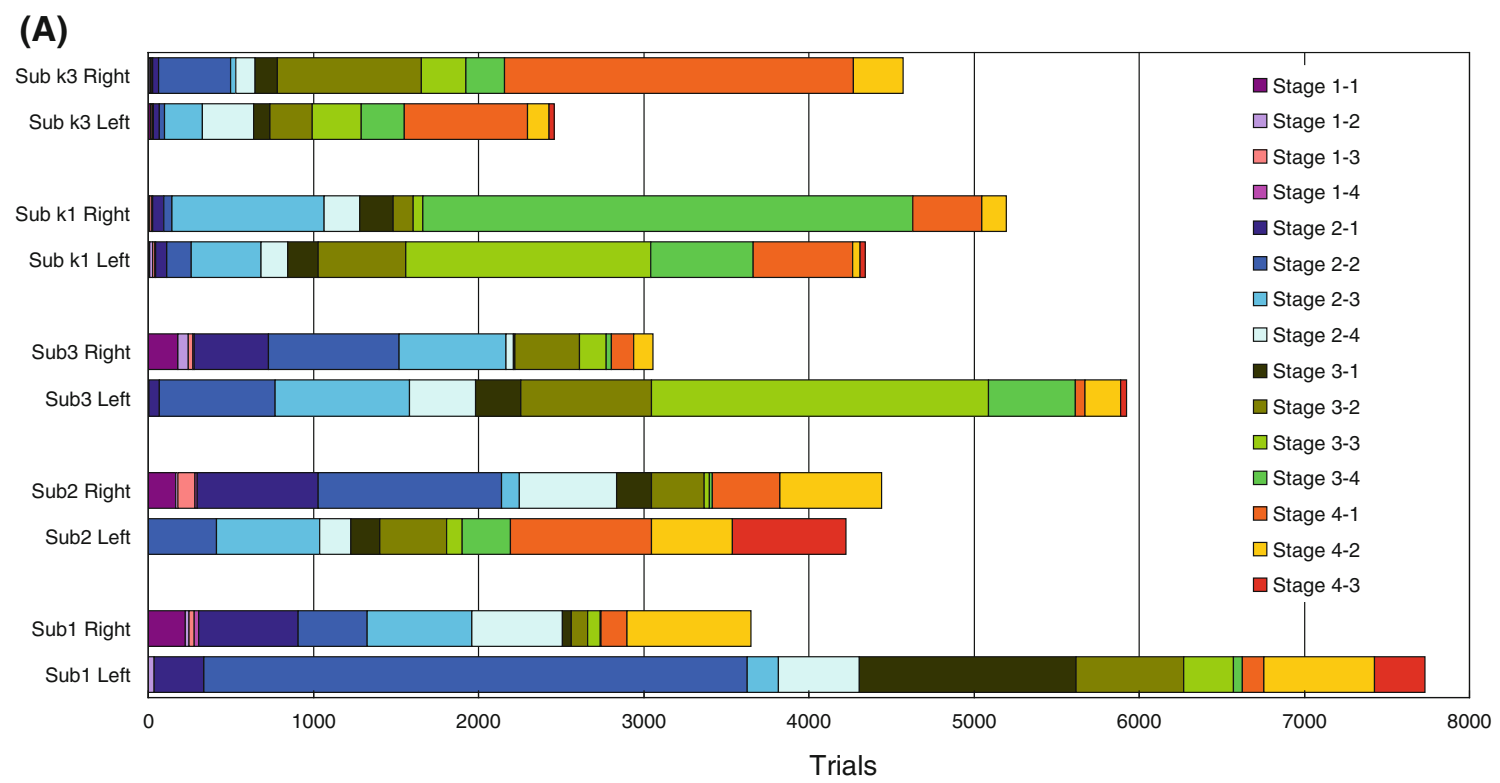

(B)
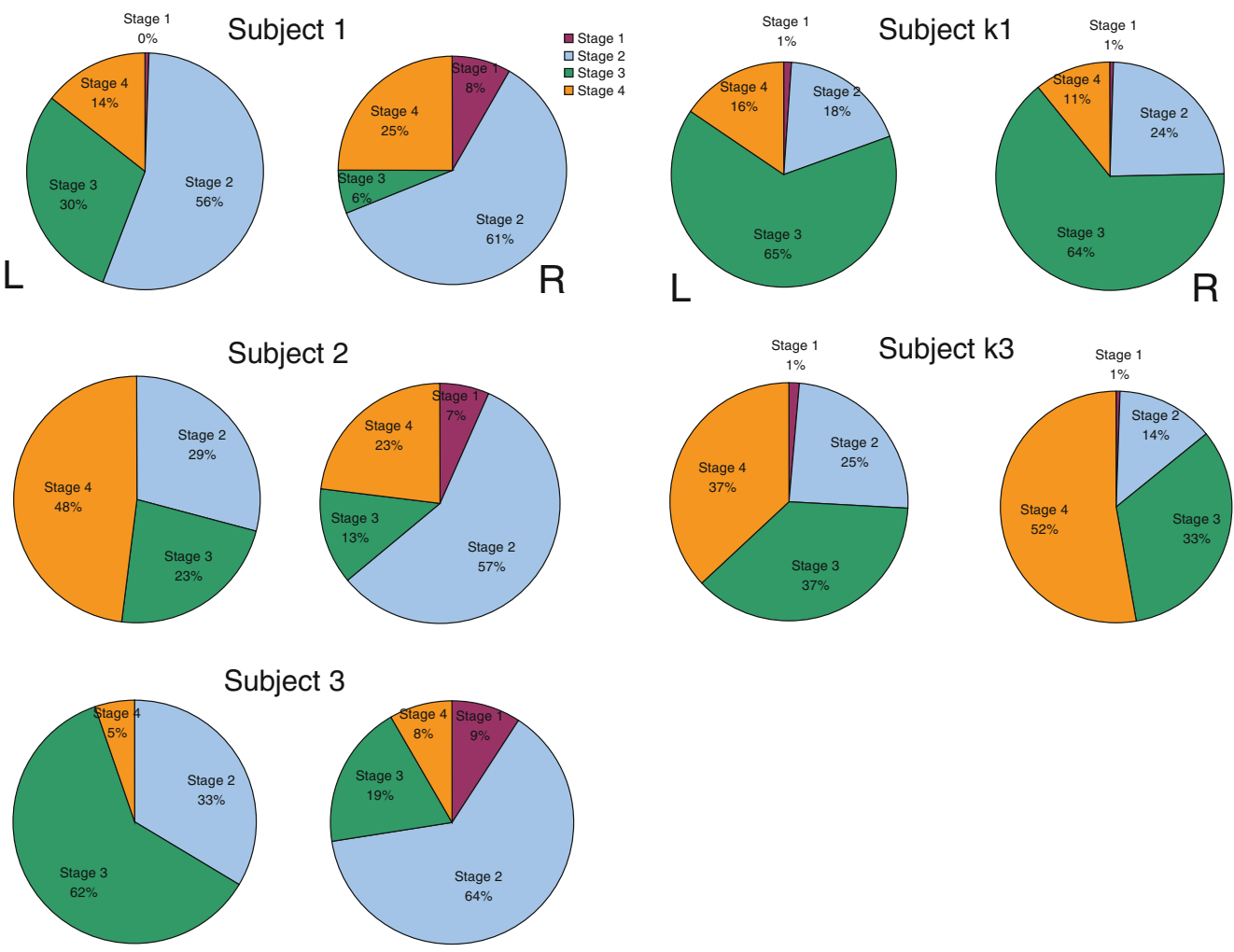

Fig. 3 a The number of trials required to reach the acquisition criterion in each step. Left or right after the subject names denotes the side on which the reward was placed relative to the rake-shaped tool. The final step of the training (Stage 4-3) was included on the left bar in each subject. Because of the change in the training protocol, only the

data from step 2 on the left side were included in Stage 1 for the left side condition in subjects 1 and 3, and the data were not included for subject 2. b Percentage of trials required to reach the criterion in each stage for each subject. Similar to (a), left (L) or right (R) denotes the side of the reward placement relative to the rake-shaped tool

7,026 for subjects 1, 2, 3, k1 and k3, respectively. Figure $3 \mathrm{~b}$ conditions in subjects $1, \mathrm{k} 1$ and $\mathrm{k} 3$; this was not the case for subjects 2 and 3. However, there were no clear trends of distribution among the subjects and between the training protocols (subject 1, 2 and 3 vs. subject $\mathrm{k} 1$ and $\mathrm{k} 3$ ). 

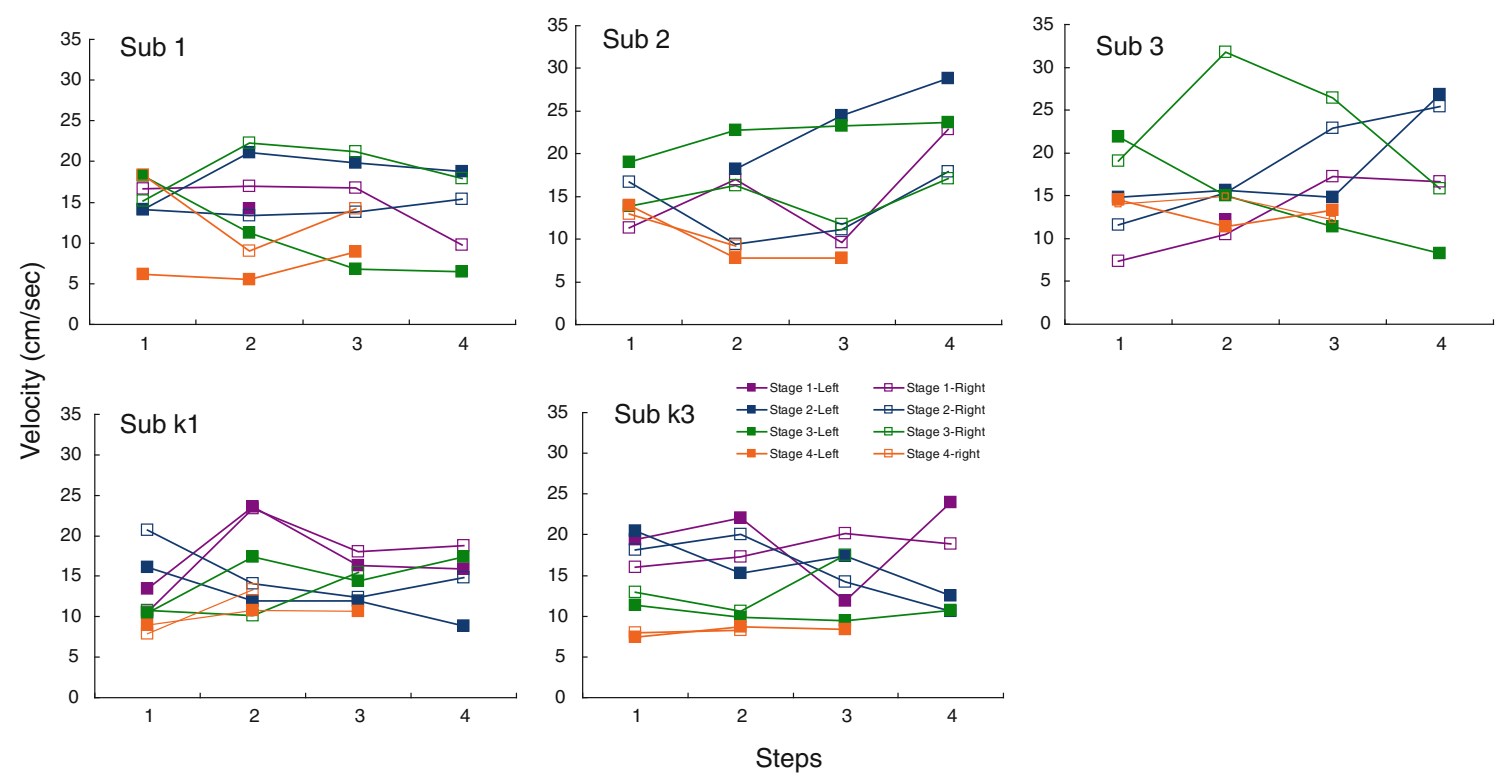

Fig. 4 Velocity $(\mathrm{cm} / \mathrm{s})$ of the tool in each step. Data were averaged for the five consecutive successful trials, which were defined as such by the acquisition criterion for a given step. Left or right denotes the position of the rewards relative to the tool

Table 2 Individual hand use in the final training step, Stage 4-3

\begin{tabular}{llllll}
\hline Subject & 1 & 2 & 3 & k1 & k3 \\
\hline Hand use in the 5 trials & Left (5/5) & Left $\rightarrow$ right $(5 / 5)$ & Right $\rightarrow$ left $(5 / 5)$ & Left $\rightarrow$ right $(5 / 5)$ & Left $\rightarrow$ right $(5 / 5)$ \\
\hline
\end{tabular}

The subject's speed with the tool increased and became more stable as they passed through the training stages, as shown in Fig. 4. A two-way ANOVA [Stage (4) $\times$ Position (left or right)] revealed a significant effect of the stages $[F(3,126)=9.673, P<0.001]$. A multiple comparison analysis (Fisher's LSD test) indicated that the speed at Stage 4 was slower than the other three stages $(P<0.001)$. There were no other effects of position $[F(1,126)=0.141$, $P=0.708)$ or interactions $[F(3,126)=2.134, P=0.099)$.

There was no clear handedness preference in our subjects. While subject 1 consistently used unilateral hand movements, the other subjects $(n=4)$ used both hands to move the tool depending on the location of the food item. That is, when the food reward was located to the left of the tool, they always used their right hand to manipulate it and vice versa. In Stage 4-3, the final training step, all of the subjects except for subject 1 used one hand to displace the tool first and then used the other hand to retrieve the food items (Table 2). Subjects 2, k1 and k3 used the left hand first and then the right hand; subject 3 used the opposite orientation (see SI video 1, 2 and 3 for videos of the performance by subject 1, 3 and $\mathrm{k} 1$ ). Finally, the subjects learned to retrieve the food wherever the food items were placed on the table (see SI video 4 for the performance by subject 3).

\section{Discussion}

In this study, common marmosets were successfully trained to manipulate a rake-shaped tool after numerous trials. This is the first time that this has been reported in this species. This finding indicates that marmosets have a high learning ability. A parametric analysis of the velocity of the tool tip (Fig. 4) confirmed the gradual change in their motor control. Successful acquisition of tool use further supports the presence of the cognitive and physical ability of these animals to use the concept of support from both production (tool-use learning) and recognition (discrimination of support) perspectives.

When comparing common marmosets to Japanese monkeys, the following three critical points have to be considered. First, there is a difference in hand structure between the common marmosets and the Japanese macaques. While macaques have nails on all of their fingers, marmosets have claws (Soligo and Müller 1999). This may considerably reduce their dexterity for tool manipulation. Thus, we modified the rake-shaped tool that had been used with Japanese macaques, creating a special handle that attached to the shaft of the tool, so that the common marmosets did not need to use a precision grip to grasp the handle. Second, there are some different features in the cortical hand enervation 
between the two species. While humans and macaques have cortico-motoneuronal projections, there is no such projection in common marmosets (Lemon and Griffiths 2005). Additionally, the gene expression of secreted phosphoprotein 1 (SPP1), which is thought to correlate with dexterous motor control in primates, is distributed in the thalamus, brainstem and spinal cord of both rhesus macaques and common marmosets; however, SPP1 distribution in the sensorimotor cortex was found in macaques but not marmosets (Higo et al. 2010). Third, macaques may use their hands more often than marmosets. In the wild, marmosets depend on plant exudates and gums heavily (20-70\% of their total intake) for their daily food intake (Ferrari 1993). When feeding, they pierce the trunks of trees and lick the exudates directly (Stevenson and Rylands 1988), without using their hands. In addition, captive marmosets often eat food pellets without using their hands.

These differences may influence the speed of learning. Compared with the results obtained with a Japanese macaque (Yamazaki et al. 2009), the marmosets required many more trials to achieve the acquisition criterion. Our marmosets required 7,026 trials at minimum (subject k3, Fig. 3a); one Japanese macaque required only 513 trials to achieve the final step of the training protocol (which was comparable to the number of trials in the present study; Rake I-VII in Yamazaki et al. 2009). In addition, the number of trials executed in a daily session was very different for the marmosets and the Japanese macaques. While the macaques executed 100-500 trials on average (Yamazaki et al. 2009), the marmosets only executed around 40 trials and sometimes even fewer in a daily session. This difference may partly be responsible for the slow skill acquisition in marmosets. We used two different training orders in Stages 1 and 2. In these stages, subjects 1, 2 and 3 learned to master the conditions with the food items on the right first, whereas subject k1 and k3 learned both sides simultaneously. The difference between these two training orders is reflected in the percentage of required trials, as shown in Fig. $3 \mathrm{~b}$. In the case of subjects $\mathrm{k} 1$ and $\mathrm{k} 3$, the required trials for mastering a given stage were similar for both sides, although there was an individual difference between the two subjects. This was not the case with subjects 1, 2 and 3 . The training protocol for subjects $\mathrm{k} 1$ and $\mathrm{k} 3$ is therefore felt to be more effective than the protocol for subjects 1, 2 and 3 because it likely enhanced the transfer of performance between the left and right sides.

With the exception of subject 1 , all of the marmosets manipulated the tools bimanually. This also differed from the macaques' performance, which showed an individual hand preference as the training proceeded (e.g. Yamazaki et al. 2009). This lack of hand preference was also different from the results of the studies using prosimians (e.g. Forsythe and Ward 1988), orangutans (Rogers and Kaplan 1996) and chimpanzees (e.g. Hopkins et al. 2005), all of which showed a population bias for a left- or right-handed preference during engaging activities, such as grasping food, touching faces and tool use. The marmosets preferred adduction to abduction when they made an arc to retrieve the food items, resulting in bimanual tool use that depended on the spatial relationship between the tool and the food. However, in Stage 4-3 where there were no position biases for the tool and the food item (because the food item and tool were placed in a row), the marmosets showed consistent tendencies in hand use (Table 2). Our results are consistent with previous findings on hand use in common marmosets, which confirmed the lack of a population bias even when the task demand was increased and became complex. However, the increased task complexity affected the degree of hand preference in each subject (Hook and Rogers 2008).

Despite several differences listed above, the kinematic change in tool-use learning was similar to that of Japanese macaques. In Ishibashi et al. (2000), the trajectory of the tool head became circular, and the velocity became more stable, without a rapid increase before impacting the food item, as tool-use training proceeded. Our parametric analysis also indicated that the velocity of the tool tip slowed and stabilised in the final stage of the training. In addition, tool tip movement became smooth, as shown in the video clips (See SI videos 1-4). As with Japanese macaques, it is plausible that some neurological features, such as extension of the axon (Hihara et al. 2006), expression of brain-derived neurotrophic factors (Ishibashi et al. 2002) and volume changes in some brain regions (Quallo et al. 2009), may contribute to the learning a novel behaviour. Thus, the fact that common marmosets learned to use the rake-shaped tool paves the way for future enquiries to examine the biological mechanisms underlying cognitive abilities for tool use at the molecular and genetic levels, using this primate species.

Acknowledgments The study was supported by the Centre for Advanced Research on Logic and Sensibility, The Global COE Program, at Keio University, Japan, and the Funding Program for World-leading Innovative R\&D on Science and Technology, at RIKEN, Japan.

Conflict of interest The authors declare that they have no conflict of interest.

Open Access This article is distributed under the terms of the Creative Commons Attribution Noncommercial License which permits any noncommercial use, distribution, and reproduction in any medium, provided the original author(s) and source are credited.

\section{References}

Asano T (1994) Tool using behavior and language in primates. In: Hayes SC, Hayes LJ, Sato M, Ono K (eds) Behavior analysis of language and cognition. Context, Reno, pp 145-148 
Ferrari SF (1993) Ecological differentiation in the Callitrichidae. In: Rylands AB (ed) Marmosets and tamarins: systematics, behaviour, and ecology. Oxford University Press, Oxford, pp 314-328

Forsythe C, Ward JP (1988) Black Lemur (L. macaco) hand preference in food reaching. Primates 29:369-374

Fragaszy D, Izar P, Visalberghi E, Ottoni EB, de Oliveira MG (2004) Wild capuchin monkeys (C. libidinosus) use anvils and stone pounding tools. Am J Primatol 64:359-366

Glazko GV, Nei M (2003) Estimation of divergence times for major lineages of primate species. Mol Biol Evol 20:424-434

Higo N, Sato A, Yamamoto T, Nishimura Y, Oishi T, Murata Y, Onoe $\mathrm{H}$, Yoshino-Saito K, Tsuboi F, Takahashi M, Isa T, Kojima T (2010) SPP1 is expressed in corticospinal neurons of the macaque sensorimotor cortex. J Comp Neurol 518:2633-2644

Hihara S, Obayashi S, Tanaka M, Iriki A (2003) Rapid learning of sequential tool use by macaque monkeys. Physiol Behav 78:427-434

Hihara S, Notoya T, Tanaka M, Ichinose S, Ojima H, Obayashi S, Fujii N, Iriki A (2006) Extension of corticocortical afferents into the anterior bank of the intraparietal sulcus by tool-use training in adult monkeys. Neuropsychologia 44:2636-2646

Hook MA, Rogers LJ (2008) Visuospatial reaching preferences of common marmosets (C. jacchus): an assessment of individual biases across a variety of tasks. J Comp Psychol 122:41-51

Hopkins WD, Russell J, Freeman H, Buehler N, Reynolds E, Schapiro SJ (2005) The distribution and development of handedness for manual gestures in captive chimpanzees (P. troglodytes). Psychol Sci 16:487-493

Hunt GR (1996) Manufacture and use of hook-tools by new Caledonian crows. Nature 379:249-251

Iriki A, Tanaka M, Iwamura Y (1996) Coding of modified body schema during tool use by macaque postcentral neurones. Neuro Rep 7:2325-2330

Ishibashi H, Hihara S, Iriki A (2000) Acquisition and development of monkey tool-use: behavioral and kinematic analyses. Can J Physiol Pharmacol 78:958-966

Ishibashi H, Hihara S, Takahashi M, Heike T, Yokota T, Iriki A (2002) Tool-use learning selectively induces expression of brain-derived neurotrophic factor, its receptor trkB, and neurotrophin 3 in the intraparietal multisensory cortex of monkeys. Cogn Brain Res $14: 3-9$
Leca JB, Gunst N, Huffman MA (2010) The first case of dental flossing by a Japanese macaque (M. fuscata): implications for the determinants of behavioral innovation and the constraints on social transmission. J Primatol 51:13-22

Lemon RN, Griffiths J (2005) Comparing the function of the corticospinal system in different species: organizational differences for motor specialization? Muscle Nerv 32:261-279

Malaivijitnond S, Lekprayoon C, Tandavanittj N, Panha S, Cheewatham C, Hamada Y (2007) Stone-tool usage by thai long-tailed macaques (M. fascicularis). Am J Primatol 69:227-233

Matsuzawa T (1994) Field experiments on the use of stone tools by chimpanzees in the wild. In: Wrangham RW, McGrew WC, de Waal FBM, Heltne PG (eds) Chimpanzee cultures. Harvard University Press, Cambridge, pp 351-370

Moura AC, Lee PC (2004) Capuchin stone tool use in caatinga dry forest. Science 306:1909

Quallo MM, Price CJ, Ueno K, Asamizuya T, Cheng K, Lemon RN, Iriki A (2009) Gray and white matter changes associated with tool-use learning in macaque monkeys. Proc Natl Acad Sci USA 106:18379-18384

Rogers LJ, Kaplan G (1996) Koehler and tool-use in orang-utans. Int J Comp Psychol 6:234-241

Sasaki E, Suemizu H, Shimada A, Hanazawa K, Oiwa R, Kamioka M, Tomioka I, Sotomaru Y, Hirakawa R, Eto T, Shiozawa S, Maeda T, Ito M, Ito R, Kito C, Yagihashi C, Kawai K, Miyoshi H, Tanioka Y, Tamaoki N, Habu S, Okano H, Nomura T (2009) Generation of transgenic non-human primates with germline transmission. Nature 459:523-527

Soligo C, Müller AE (1999) Nails and claws in primate evolution. J Hum Evol 36:97-114

Stevenson MF, Rylands AB (1988) The marmosets, genus Callithrix. In: Mittermeier RA, Cimbra-Filho A, Fonseca GAB (eds) Ecology and behavior of neotropical primates, vol 2. World Wildlife Fund, Washington DC, pp 131-222

Yamazaki Y, Namba H, Iriki A (2009) Acquisition of an externalized eye by Japanese monkeys. Exp Brain Res 194:131-142

Yamazaki Y, Iriki A, Watanabe S (2011) Modulation of physical understanding by common marmosets (C. jacchus). Anim Cogn $14: 175-186$ 